\title{
SYNERGISTIC ACTIVITY OF ASTROMICIN AND $\beta$-LACTAM ANTIBIOTICS AGAINST PSEUDOMONAS AERUGINOSA IN VITRO AND IN VIVO
}

\author{
Kozo Kitaura, Kazuyuki Mineura, Nobuhiro Nakamizo, Khoya Shiba ${ }^{\dagger}$, \\ MASAHISA OHMORI ${ }^{\dagger}$, Atsushi SAIto ${ }^{\dagger}$ and Yasushi Ueda ${ }^{\dagger}$ \\ Pharmaceutical Research Laboratory, Kyowa Hakko Kogyo Co., Ltd. \\ Mishima, Japan \\ †The Second Department of Internal Medicine, School of Medicine, The Jikei University, \\ Tokyo, Japan
}

(Received for publication May 14, 1984)

\begin{abstract}
Synergistic activity of astromicin and an antipseudomonal $\beta$-lactam antibiotic such as piperacillin, cefsulodin or carbenicillin against Pseudomonas aeruginosa was demonstrated in vitro and in vivo.

Synergy in vitro was observed more often when astromicin was combined with piperacillin or cefsulodin than when it was combined with carbenicillin. The combination of astromicin with piperacillin showed a bactericidal activity against Pseudomonas aeruginosa at a bacteriostatic concentration of each antibiotic alone.

The synergy observed in vitro was reproduced against experimental mouse infections, and the astromicin-piperacillin or cefsulodin combination produced significantly greater protective effects than the single use of individual antibiotics.
\end{abstract}

The incidence of Pseudomonas aeruginosa infections has shown an increase in recent years, and it is well recognized that $\boldsymbol{P}$. aeruginosa is among the most resistant microorganisms to commonly used antibiotics $^{1)}$.

Serious infections due to $P$. aeruginosa are often treated with a combination of an aminoglycoside antibiotic and an antipseudomonal $\beta$-lactam antibiotic such as carbenicillin or sulbenicillin to achieve additive or synergistic antibacterial activity.

Astromicin (ASTM)*, an aminoglycoside antibiotic containing the novel aminocyclitol and fortamine, is highly active in vitro and in vivo against most Gram-positive and Gram-negative bacteria ${ }^{2 \sim 4)}$. This antibiotic is characterized by stability to aminoglycoside-inactivating enzymes except AAC(III) $-1^{2)}$, and low-grade oto- and nephro-toxicity ${ }^{5)}$. It shows low activity against $P$. aeruginosa however ${ }^{3,4}$.

The present report describes a synergistic relationship of astromicin with $\beta$-lactam antibiotics against $P$. aeruginosa strains both in vitro and in vivo.

\section{Materials and Methods}

Antibiotics

Piperacillin (PIPC) was purchased from Toyama Chemical Co., Ltd.; cefsulodin (CFS) and carbenicillin (CBPC) were from Takeda Pharmaceuticals Co., Ltd. ASTM was prepared in Kyowa Hakko Kogyo Co., Ltd.

Organisms

The laboratory strains were maintained on heart infusion agar (HIA, Eiken Chemicals, Tokyo).

* Previously, astromicin was called fortimicin A. 
Clinical isolates were kindly supplied by several clinical laboratories and maintained in cooked meat medium (Nissui Seiyaku Co., Ltd., Tokyo).

Antibiotic Susceptibility Testing

MICs of antibiotics were measured by the two-fold serial dilution method using HIA plates with an inoculum of $10^{8}$ colony forming units (cfu). The MIC values $(\mu \mathrm{g} / \mathrm{ml})$ were read after 18 hours of incubation at $37^{\circ} \mathrm{C}$.

\section{Synergy In Vitro}

The MICs of ASTM in combination with each $\beta$-lactam antibiotic against 27 strains of $P$. aeruginosa were determined by the checkerboard method using HIA at an inoculum of $10^{8} \mathrm{cfu}$. After 18 hours of incubation at $37^{\circ} \mathrm{C}$, the MICs of the $\beta$-lactam antibiotic and ASTM used alone or in combination were defined by no visible growth on plates.

The fractional inhibitory concentration (FIC) index was calculated by dividing the MIC obtained for each of the components in the combination by that for each component alone and adding the two quotients ${ }^{6}$. Criteria for the effects of combination were defined as follows:

1) Synergy - the index was $\leq 0.5$

2) Partial synergy - the index was $>0.5 \sim 0.75$

3) Indifferent - the index was $>0.75 \sim 1.0$

4) Antagonism - the index was $>1.0$

Bactericidal Activity

Rates of killing of two $P$. aeruginosa strains by ASTM alone and in combination were measured in heart infusion broth (Difco Laboratories, U.S.A.) using a fresh dilution of organisms from an overnight culture in Trypticase soy broth (Nissui Seiyaku Co., Ltd., Tokyo). After incubation for 2 hours at $37^{\circ} \mathrm{C}$ with shaking, serial two-fold dilutions of ASTM and PIPC were added and samples of culture were taken at selected intervals, immediately diluted in broth, and plated on HIA. The cfu was counted after overnight incubation.

Synergy In Vivo

Male $d d \mathrm{Y}$ mice, weighing 18 to $20 \mathrm{~g}$, were infected intraperitoneally with suspensions of test organism in $5 \%$ hog gastric mucin (ICN Pharmaceuticals, Inc., U.S.A.) in a volume of $0.5 \mathrm{ml}$. The challenge dose was approximately minimal lethal doses (inoculum size: $0.7 \times 10^{4} \sim 0.5 \times 10^{8}$ cells/ mouse). The antibiotics were administered subcutaneously to a group of 10 mice 2 hours after infection. Based on the survival rate on the 7 th day of infection, the $50 \%$ effective dose $\left(\mathrm{ED}_{50}\right)$ and its confidence limits were calculated by the LITCHFIELD-WILCOXON method ${ }^{7)}$. The extent of synergy of a combination was expressed as the synergistic ratio $(\mathrm{SR})^{8, \theta)}$. The SR, which corresponds to the FIC index, is a ratio of experimentally determined potency of the combined drugs over a hypothetical potency in which additive effect of both drugs is assumed. Synergy in vivo was considered to have occurred if the lowest $95 \%$ confidence limit of the SR was greater than 1.0 .

\section{Results}

Antibacterial Activity In Vitro

In vitro susceptibilities of 27 strains of $P$. aeruginosa to ASTM, PIPC, CFS and CBPC are shown in Fig. 1.

ASTM inhibited 15 of 27 strains of $P$. aeruginosa at a concentration of $25 \mu \mathrm{g} / \mathrm{ml}$. It was 2 to 4 times more active than CBPC, slightly less active than PIPC, but much less active than CFS. CFS was the most active of the four antibiotics against strains of $P$. aeruginosa, with an $\mathrm{MIC}_{50} \leq 6.25 \mu \mathrm{g} / \mathrm{ml}$.

\section{Synergy In Vitro}

The FIC indices of the combination of ASTM and $\beta$-lactam antibiotics using the checkerboard titration method on 27 strains of $P$. aeruginosa are summarized in Table 1. 
Fig. 1. Susceptibility of 27 strains of $P$. aeruginosa.

$\square$ Cefsulodin, $\triangle$ piperacillin, $\bigcirc$ astromicin, $\Delta$ carbenicillin.

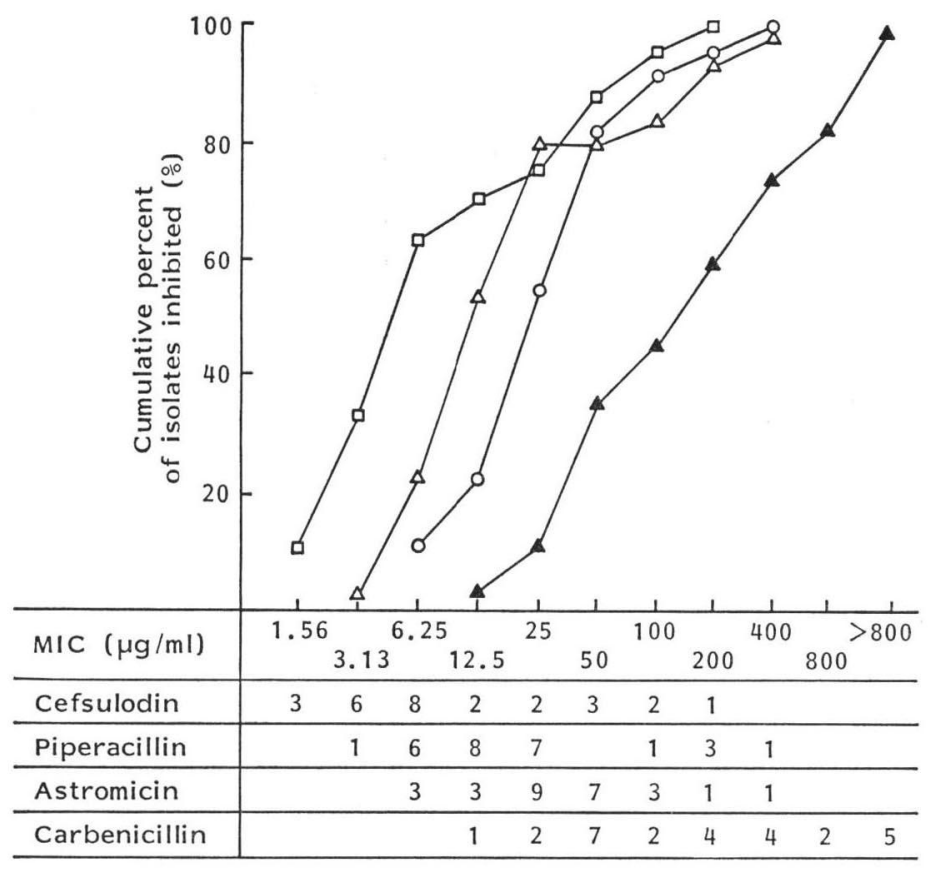

Table 1. Combinations of ASTM with PIPC, CFS or CBPC against $P$. aeruginosa (27 strains).

\begin{tabular}{|c|c|c|c|c|}
\hline \multirow{2}{*}{ Combination } & \multicolumn{4}{|c|}{ No. of strains $(\%)$} \\
\hline & Synergy & Partial synergy & Indifferent & Antagonism \\
\hline ASTM + PIPC & $\begin{array}{c}17 \\
(63.0)\end{array}$ & $\begin{array}{l}10 \\
(37.0)\end{array}$ & 0 & 0 \\
\hline $\mathrm{ASTM}+\mathrm{CFS}$ & $\begin{array}{c}15 \\
(55.6)\end{array}$ & $\begin{array}{c}9 \\
(33.3)\end{array}$ & $\begin{array}{l}2 \\
(7.4)\end{array}$ & $\begin{array}{l}1 \\
(3.7)\end{array}$ \\
\hline ASTM + CBPC* & $\begin{array}{c}8 \\
(29.6)\end{array}$ & $\begin{array}{c}15 \\
(55.6)\end{array}$ & $\begin{array}{c}2 \\
(7.4)\end{array}$ & $\begin{array}{c}1 \\
(3.7)\end{array}$ \\
\hline
\end{tabular}

* FIC index of one strain was not determined.

The ASTM-PIPC combination was most synergistic. A synergistic effect was observed more often when ASTM was combined with PIPC (17 strains, 63.0\%) or CFS (15 strains, 55.6\%) than when it was combined with CBPC ( 8 strains, $29.6 \%$ ). Synergy plus partial synergy were produced against all strains by the ASTM-PIPC combination, against $24(88.9 \%)$ and $23(85.2 \%)$ by the ASTM-CFS and ASTM-CBPC combinations, respectively.

\section{Bactericidal Effect}

The comparative growth of $P$. aeruginosa F-1997 and E-2 in the presence of ASTM or PIPC alone and in combination are shown in Fig. 2.

Against $P$. aeruginosa strain F-1997, the addition of a sub-lethal concentration of PIPC resulted in a marked increase in the rate of bacterial killing of ASTM. Against P. aeruginosa strain E-2, the rate of bacterial killing was also clearly enhanced by the drug combination. 
Fig. 2. Comparative growth curves of $P$. aeruginosa strain F-1997 (A) and strain E-2 (B) in the presence of ASTM or PIPC alone and in combination.

- Control, $\bigcirc$ astromicin $3.13 \mu \mathrm{g} / \mathrm{ml}, \Delta$ piperacillin $3.13 \mu \mathrm{g} / \mathrm{ml}$, $\mathbf{a}$ astromicin $3.13 \mu \mathrm{g} / \mathrm{ml}+$ piperacillin $3.13 \mu \mathrm{g} / \mathrm{ml}$.

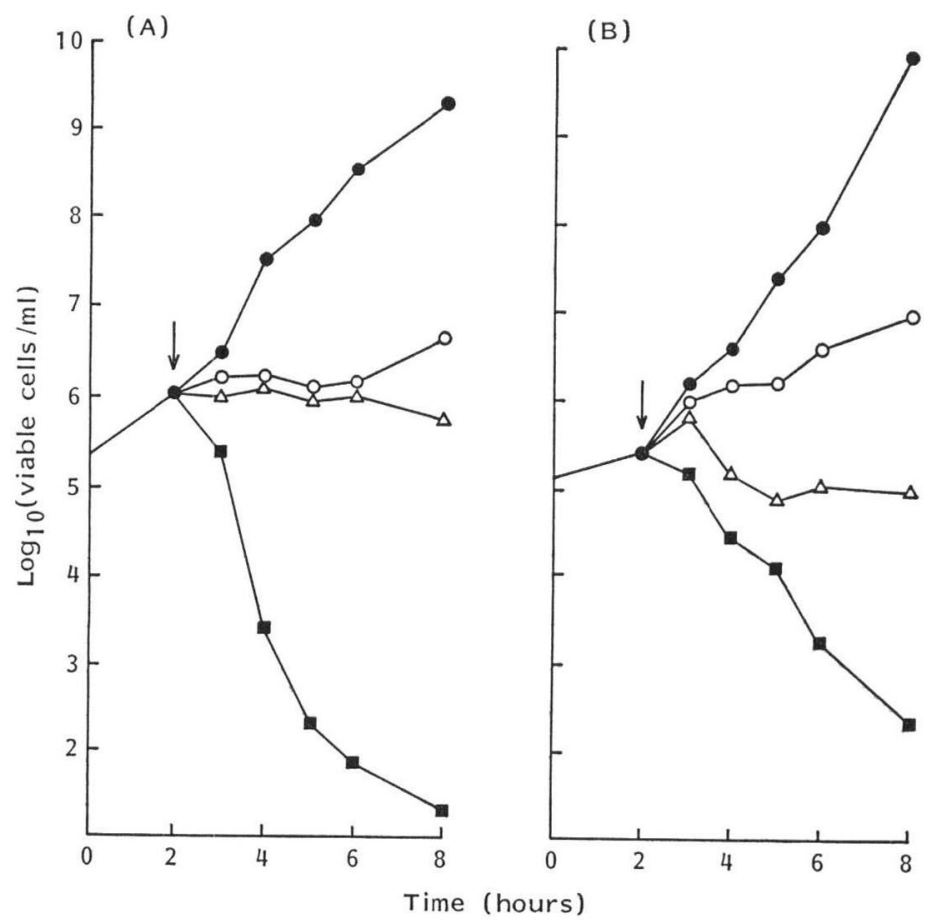

Table 2. Synergistic effect of ASTM, PIPC or CFS against intraperitoneal $P$. aeruginosa injection in mice.

\begin{tabular}{|c|c|c|c|c|c|c|}
\hline $\begin{array}{c}\text { Strain* \& } \\
\text { inoculum } \\
\text { (cells/mouse) }\end{array}$ & $\begin{array}{l}\text { FIC } \\
\text { index }\end{array}$ & $\begin{array}{c}\text { Antibiotic** } \\
(\mathrm{MIC}, \mu \mathrm{g} / \mathrm{ml})\end{array}$ & $\begin{array}{l}\text { Combina- } \\
\text { tion } \\
\text { ratio }\end{array}$ & $\begin{array}{c}\mathrm{ED}_{50} \\
(\mathrm{mg} / \mathrm{kg})\end{array}$ & $\begin{array}{l}\text { Syner- } \\
\text { gistic } \\
\text { ratio }\end{array}$ & $\begin{array}{c}\text { Confidence } \\
\text { limits } \\
(P=0.05)\end{array}$ \\
\hline $\begin{array}{l}\text { F-2043 } \\
0.5 \times 10^{8}\end{array}$ & 0.19 & $\begin{array}{l}\text { ASTM }(100) \\
\text { PIPC }(200) \\
\text { ASTM+PIPC }\end{array}$ & $\begin{array}{l}1: 5 \\
1: 10\end{array}$ & $\begin{array}{c}29.3 \\
7,060 \\
80.3 \\
95.8\end{array}$ & $\begin{array}{l}2.1 \\
3.2\end{array}$ & $\begin{array}{l}1.4 \sim 3.2 \\
2.1 \sim 5.0\end{array}$ \\
\hline $\begin{array}{l}\text { F-1997 } \\
0.7 \times 10^{4}\end{array}$ & 0.16 & $\begin{array}{l}\text { ASTM (100) } \\
\text { PIPC (25) } \\
\text { ASTM + PIPC }\end{array}$ & $\begin{array}{l}1: 5 \\
1: 10\end{array}$ & $\begin{array}{l}90.3 \\
639 \\
143 \\
208\end{array}$ & $\begin{array}{l}2.2 \\
2.0\end{array}$ & $\begin{array}{l}1.5 \sim 3.2 \\
1.4 \sim 2.8\end{array}$ \\
\hline $\begin{array}{l}\text { E-2 } \\
0.8 \times 10^{5}\end{array}$ & 0.38 & $\begin{array}{l}\text { ASTM (25) } \\
\text { PIPC }(6.25) \\
\text { ASTM + PIPC }\end{array}$ & $\begin{array}{l}1: 5 \\
1: 10\end{array}$ & $\begin{array}{r}208 \\
2,868 \\
446 \\
740\end{array}$ & $\begin{array}{l}2.1 \\
1.8\end{array}$ & $\begin{array}{l}1.2 \sim 3.5 \\
1.0 \sim 3.1\end{array}$ \\
\hline $\begin{array}{l}\text { KY }-8512 \\
0.5 \times 10^{8}\end{array}$ & 0.38 & $\begin{array}{l}\text { ASTM (12.5) } \\
\text { CFS }(6.25) \\
\text { ASTM }+ \text { CFS }\end{array}$ & $\begin{array}{l}1: 1 \\
1: 5 \\
1: 10\end{array}$ & $\begin{array}{c}137 \\
297 \\
50.5 \\
167 \\
140\end{array}$ & $\begin{array}{l}3.7 \\
1.5 \\
1.9\end{array}$ & $\begin{array}{l}1.4 \sim 9.6 \\
0.6 \sim 3.7 \\
0.8 \sim 4.7\end{array}$ \\
\hline
\end{tabular}

* P. aeruginosa was inoculated with $5 \%$ mucin.

** Antibiotic was administered subcutaneously 2 hours after injection. 


\section{Synergy In Vivo}

To ascertain further the effect of combinations in vivo, the efficacy of ASTM, PIPC or CFS alone and in combination, was measured against murine peritoneal infections due to $P$. aeruginosa. In Table 2 the results of in vivo tests performed with 4 strains of $P$. aeruginosa are summarized. The $\mathrm{ED}_{50}$ values of the ASTM-PIPC combination at a ratio of 1:5 or 1:10 were definitely decreased as compared with that of each antibiotic alone.

The combination showed synergistic activity with SR values of 1.8 to 3.2 against all 3 strains of $P$. aeruginosa tested.

On the other hand, the ASTM-CFS combination at a ratio of $1: 5$ or $1: 10$ resulted in an additive effect against infection due to strain KY-8512. A synergistic effect was observed at a ratio of 1:1 with this combination however.

These results support the view that the synergistic effect in fact occurs in vivo as well as in vitro.

\section{Discussion}

Various combinations of antibiotics and chemotherapeutic agents are now used to obtain an extended antibacterial spectrum, to increase antibacterial activity and to prevent the appearance of resistant bacteria. ASTM, a new aminoglycoside antibiotic, has antimicrobial activity similar to that of amikacin, but lacks significant activity against $P$. aeruginosa ${ }^{3,4,10)}$. It has been found previously however that there is often a synergistic antimicrobial effect between aminoglycosides and $\beta$ lactams $^{11 \sim 14)}$.

The results of experiments reported here show that the combination of ASTM with antipseudomonal $\beta$-lactam antibiotics exhibited partially synergistic or synergistic activity against most strains of $P$. aeruginosa tested. In particular, the ASTM-PIPC combination showed partially synergistic or synergistic activity against all strains tested as well as strong bactericidal activity at a bacteriostatic concentration of each antibiotic alone. The synergy observed in vitro was reproduced against experimental mouse infections, and ASTM-PIPC or ASTM-CFS combinations produced a significantly greater protective effect than the individual antibiotics. Recently, YAmASHITA et al. ${ }^{15)}$ reported that the combination of ASTM with $\beta$-lactam antibiotics such as CBPC or PIPC may act synergistically in vitro against $P$. aeruginosa including ASTM- and GM-resistant strains.

The present studies report the first demonstration of ASTM-PIPC and ASTM-CFS synergy in vivo for infections caused by isolates of $P$. aeruginosa, against which these antibiotics are synergistic in vitro, and provide experimental support for further clinical trials. Taking into consideration that peak serum levels achieved after $200 \mathrm{mg}$ intramuscular injection of ASTM in patients were around $12 \mu \mathrm{g} / \mathrm{ml}$, this antibiotic combination therapy seems promising.

\section{References}

1) Lowbury, E. J. L. \& R. J. Jones: Treatment and prophylaxis for Pseudomonas infections. In Resistance of Pseudomonas aeruginosa. Ed. M. R. W. Brown, pp. 237 248, John Wiley \& Sons, Inc., London, 1975

2) Nara, T.; M. Yamamoto, I. Kawamoto, K. Takayama, R. Okachi, S. Takasawa, T. Sato \& S. Sato: Fortimicins A and B, new aminoglycoside antibiotics. I. Producing organism, fermentation and biological properties of fortimicins. J. Antibiotics 30: 533 540, 1977

3) Girolami, R. L. \& J. M. Stamm: Fortimicins A and B, new aminoglycoside antibiotics. IV. In vitro study of fortimicin A compared with other aminoglycosides. J. Antibiotics 30: 564 570, 1977

4) Ohashi, Y.; H. Kawabe, K. Sato, N. Nakamura, S. Kurasige \& S. Mitsuhashi: In vitro and in vivo antibacterial activity of KW 1070, a new aminoglycoside antibiotic. Antimicrob. Agents Chemother. 17: $138 \sim 143,1980$

5) SAIto, A.; Y. Ueda \& M. Akryoshi: Experimental studies on the ototoxicity and nephrotoxicity of fortimicin A. In Current Chemotherapy and Infectious Disease, Proceedings of the 11th ICC and the 19th ICAAC. p. $401 \sim 403$, Am. Soc. Microbiol., Washington, 1980 
6) Elion, G. B.; S. Singer \& G. H. Hitchings: Antagonists of nucleic acid derivatives. III. Synergism in combinations of biochemically related antimetabolites. J. Biol. Chem. 208: 477 488, 1954

7) Litchfield, J. T. \& F. Wilcoxon: A simplified method of evaluating dose-effect experiments. J. Pharm. Exp. Therap. 96: 99 113, 1949

8) KaWAKami, M.; Y. Nagai, S. Shimizu \& S. Mitsuhashi: Antimicrobial effect of combinations of colistin methanesulfonate and chloramphenicol. I. In vitro effect. J. Antibiotics 24: 884 891, 1971

9) Tone, J.; S. Shioji, T. Kondo, M. Kawakami \& S. Mitsuhashi: Quantitative and qualitative determinations of the combined effect of tetracycline and oleandomycin. II. In vivo effect. Jpn. J. Antibiotics 29: $571 \sim 579,1976$

10) Thornsberry, C.; A. L. Barry, R. N. Jones, C. N. Baker, R. E. Badal \& R. R. Packer: Antibacterial activity of fortimicin A compared with those of five other aminoglycosides, and factors affecting susceptibility tests. Antimicrob. Agents Chemother. 19: 122 129, 1981

11) Klastersky, J.; B. Nyamubeya \& L. Vandenborre: Antimicrobial effectiveness of kanamycin, aminosidin, BB-K8, sisomicin, gentamicin and tobramycin combined with carbenicillin or cephalothin against Gram-negative rods. J. Med. Microbiol. 74: 465 472, 1974

12) Hyams, P. J.; M. S. Simberkoff \& J. J. Pahal, Jr.: Synergy between cephalosporin and aminoglycoside antibiotics against Providencia and Proteus. Antimicrob. Agents Chemother. 5: 571 577, 1974

13) Nakazawa, S.; T. Nishino, M. Otsuki, M. Nakao \& T. Nomura: Bacteriological studies on the combined action of aminoglycoside antibiotics and synthetic penicillins against Pseudomonas aeruginosa. J. Antibiotics 27: 989 991, 1974

14) Glew, R. H.; R. C. Moellering, Jr. \& C. Wennersten: Comparative synergistic activity of nafcillin, oxacillin, and methicillin in combination with gentamicin against enterococci. Antimicrob. Agents Chemother. 7: 828 832, 1975

15) Yamashita, K.; H. Kawabe \& S. Mitsuhashi: Synergistic activity of fortimicin A, and $\beta$-lactam antibiotics against Pseudomonas aeruginosa. Antimicrob. Agents Chemother. 20: 33 37, 1981 